\title{
Influence of Heat Transfer Process in Porous Media with Air Cavity- A CFD Analysis
}

\author{
Vijay Shankar ${ }^{1}$, Andreas Bengtson ${ }^{2}$, Victor Fransson ${ }^{2}$, Carl-Eric Hagentoft ${ }^{2}$ \\ ${ }^{1}$ Luleå University of Technology \\ Luleå, Sweden \\ vijay.shankar@1tu.se \\ ${ }^{2}$ Chalmers University of Technology \\ Gothenburg, Sweden
}

\begin{abstract}
Investigating the heat transfer in porous media is of interest, since a deeper understanding of this phenomenon can be used to improve the energy efficiency of buildings. Heat can be transferred in three ways: conduction, convection and radiation. All these three mechanisms are always present and needs to be taken into account. The forces generated by density gradients in the earth's gravitational field, leads to the so-called natural convective heat transfer, both in fluid and porous media. The presence of temperature gradients, when reaching a certain temperature difference, gives rise to fluid and thermal motion due to the natural convective process. The ability to simulate and compute the combined effects of heat transfer due to conduction, natural convection and radiation are therefore of paramount interest, in order to design the future environmentally friendly, energy efficient and healthy buildings. In this study, the heat transfer through a porous region, representing a layer of insulation, with an air cavity above has been numerically investigated with the help of CFD. The numerical results obtained are validated with experimental results.
\end{abstract}

Keywords: CFD, Heat Transfer, Porous Media, ANSYS

\section{Introduction}

The process of heat transfer has an important influence in all aspects of our lives. In the design of energy efficient buildings for sustainable development, the heat transfer processes is often the limiting factor. By means of utilizing the knowledge in Computational Fluid Dynamics (CFD) and applied heat transfer, greater knowledge of air movement and heat loss that occurs in buildings can be obtained. This knowledge can then be used to optimize and design environmentally friendly and energy efficient buildings for future sustainable development.

CFD simulations are preferable to real-life experiments in many ways, e.g.: full-scale testing in artificial climate is very time consuming, costly and very difficult to accomplish, because of the size of the facility and the very low temperatures required. Extensive literature study has shown that the basic research conducted so far have only dealt with certain aspects [4-12]. However, during the course of this state of the art research work, the total heat loss that occurs is calculated in order to estimate the global effects of heat transfer due to conduction, natural convection and radiation.
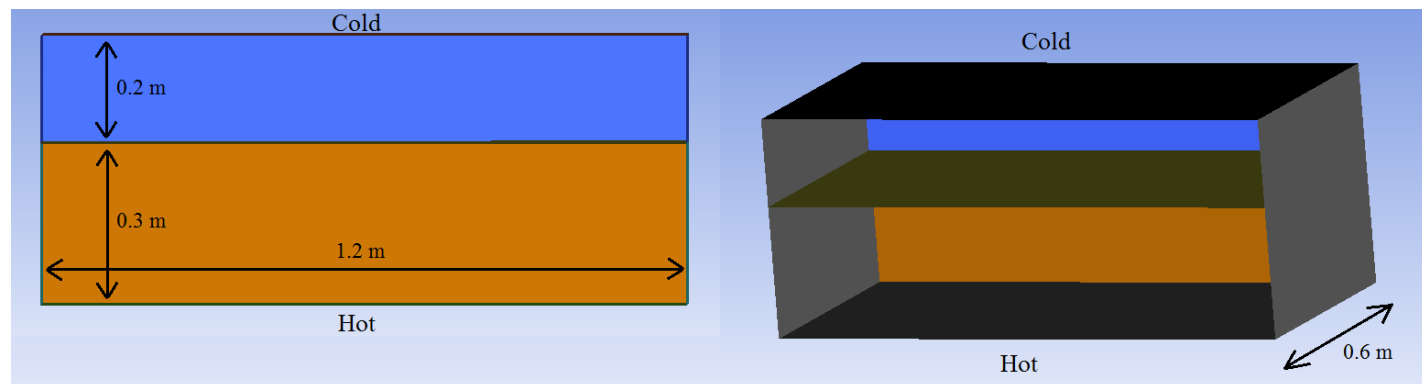

Fig. 1: The left figure shows the geometry of the two-dimensional box model, where the upper region represents the air cavity and the lower one the insulation. The figure to the right shows the three-dimensional box model, which in addition to the dimensions in the two-dimensional case has a specified thickness. 
The simulations were performed on a simple box model, which consists of a porous layer of insulation with an air layer above. The geometry of this box is identical to the geometry used in the experimental research by[7], in order to easily compare the results.

The top and bottom of the box are maintained at constant temperatures, where the temperature of the bottom always is warmer than the temperature at the top, while the sides are assumed to be adiabatic. The box is a closed system, meaning no fluid enters or exits its boundaries. Both two and three dimensional simulations were conducted, and the temperature difference between the top and bottom of the box was varied between 10 to $50 \mathrm{~K}$.

Figure 1 shows the geometry of the two and three-dimensional box models.

The CFD solver ANSYS Fluent was used for both the two and three dimensional simulations, while the computational grids were generated in ANSYS ICEM. The realizable k- $\varepsilon$ model was used to model turbulence in all simulations.

\section{Mathematical Formulation (CFD)}

The main equations that needs to be solved includes governing transport equations and equations for the turbulence model [1] as described below.

\subsection{Governing Equations}

The standard general transport equation for continuity, momentum and energy are solved [2]. For the air cavity, the realizable $\mathrm{k}-\varepsilon$ model [3] is used to model turbulence. In order to predict the heat transfer due to radiation, the P1 model is used [1]. This model solves the transport equation for radiation to determine the local radiation intensity when the P1 model is applied.

\subsection{Porosity}

The porosity [6] or void fraction, of a porous medium is defined as the fraction of the total volume that is occupied by voids. For a porous medium with porosity $\varphi$, the volume occupied by solid material is thus 1- $\varphi$.

\subsection{Porous Media in Fluent}

The presence of porous media is modeled in [2] as an extra momentum source term to the standard flow equations. This source term consists of two terms: the first one due to viscous losses and the second one due to inertial losses. The source term is defined as:

$$
S_{i}=\left(\frac{\mu}{K} v_{i}+C_{2} \frac{1}{2} \rho|v| v_{i}\right)
$$

where $\mathrm{K}$ is the permeability of the porous material and $\mathrm{C}_{2}$ is the inertial resistance factor. For laminar flows in porous media, the inertial losses are small compared to the viscous losses and $\mathrm{C}_{2}$ can be considered to be zero. The porous media model is then reduced to Darcy's law.

\section{Dimensionless Parameters}

This section presents brief descriptions of the important dimensionless parameters for this study.

\subsection{Modified Rayleigh Number}

The modified Rayleigh number is the relationship between the buoyant and the viscous forces in a fluid. It is defined as:

$$
R a_{m}=\frac{\rho \cdot c_{p} \cdot g \cdot \beta \cdot d_{m} \cdot K \cdot \Delta T}{v \cdot k_{m}}
$$

When the modified Rayleigh number exceeds a certain, critical value, natural convection is present. This is called the critical modified Rayleigh number, $R a_{m}^{*}$ 


\subsection{Nusselt Number}

The Nusselt number is defined as the ratio between heat flux with and without convection:

$$
N u=\frac{q_{\text {with convection }}}{q_{\text {without convection }}}
$$

\section{Boundary Conditions}

The applied thermal boundary conditions at the walls of the domain are prescribed temperature (Dirichlet):

$$
T=T_{b}
$$

and prescribed heat flux (Neumann):

$$
k \frac{\partial T}{\partial n}=-q_{b}
$$

where the subscript $\$ b \$$ indicates boundary. When the heat flux is prescribed to be zero, the boundary condition is called adiabatic. This simulates a perfectly insulated boundary.

\subsection{Numerical Setup}

The meshes were both constructed using hexahedronal cells only in ANSYS ICEM. The number of cells for the twodimensional case was 206142 cells and for the three dimensional case it was 475658 cells.

The simulations presented in this article were all conducted as steady simulations using the pressure-base solver in Fluent. To model the turbulence, the realizable k- $\varepsilon$ model was used. Radiation was modeled using the P1 model in Fluent [2] The pressure-velocity scheme used was SIMPLEC, and the buoyancy was modeled using the Boussinesq approximation.

The temperature of the bottom was set to $294 \mathrm{~K}$ in all simulations, while the temperature of the top was changed. In order to numerically simulate the insulation, this part of the domain was set as a porous zone in Fluent. The insulation is then modeled according to the section about porous media as described above. The permeability was set to $5 \cdot 10^{-8} \mathrm{~m}^{2}$, the porosity to 0.332 , and the thermal conductivity of the insulation to 0.044 since this was the values used in the experimental work [7] that was used for validation. In these experiments, polystyrene balls were used as insulation material.

The area with the insulation was also specified as a laminar zone, which means that the effect of turbulence is suppressed in this zone. This setting is recommended by [3] unless the permeability is very high.

\section{Results}

The results from the two and three dimensional simulations are presented in this section. In order to compute the Nusselt number in Figure 2 simulations were performed with and without flow equations (i.e. convection) enabled. The Nusselt number could then be obtained by calculating, and taking the ratio of, the heat flux in the situation with and without convection 


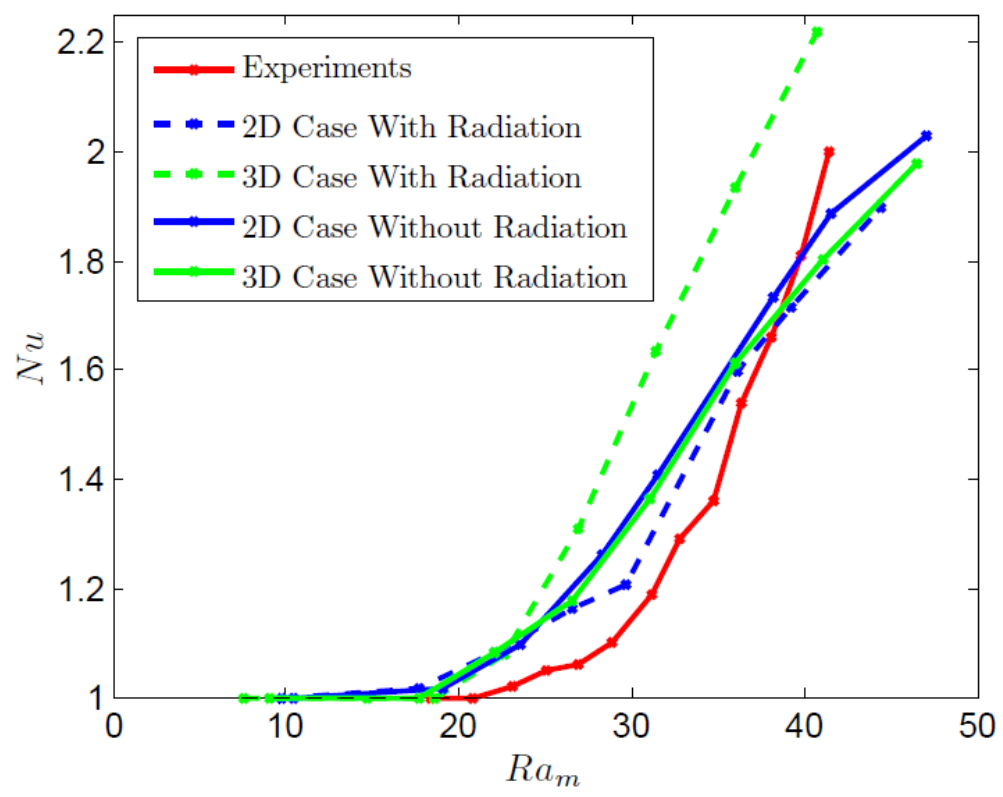

Fig. 2: Plot of the Nusselt number versus the modified Rayleigh number for the 2D case (blue line), the 3D case (green line) and the values obtained by the experimental work [7] (red line).

The onset of convection occurs at approximately $R a_{m}^{*}=16$ for the two-dimensional case, and at approximately $\mathrm{Ra}_{\mathrm{m}}=18$ for the three-dimensional case compared to approximately at $R a_{m}^{*}=21$ for the experiments. The addition of radiation had a small impact on the onset of convection.

We can infer from Figure 2 that as the temperature difference increases, the magnitude of heat transfer due to convection increases.

Also, since the three-dimensional simulations were found to be stable, the same contributes to the difference in results obtained compared to the two-dimensional case.

Computations were executed for eight temperature differences for each case, both with and without convection. The total number of simulations was thus 32 .

Due to the limited amount of space, the scales for both the temperature and vector plots can be quite hard to read. For the temperature contour plots in Figure 3 the scale in each picture goes from 294 to 294 minus the temperature difference, where red is the warmest (bottom surface) and blue the coldest (top surface). For the velocity vector plots in Figure 4 the exact velocities are not of interest, but they are in the order of $10^{-4} \mathrm{~m} / \mathrm{s}$ in the insulation and $10^{-2} \mathrm{~m} / \mathrm{s}$ in the air cavity.

The results for the three dimensional case were taken in a vertical plane in the middle of the box parallel to its longest side. 


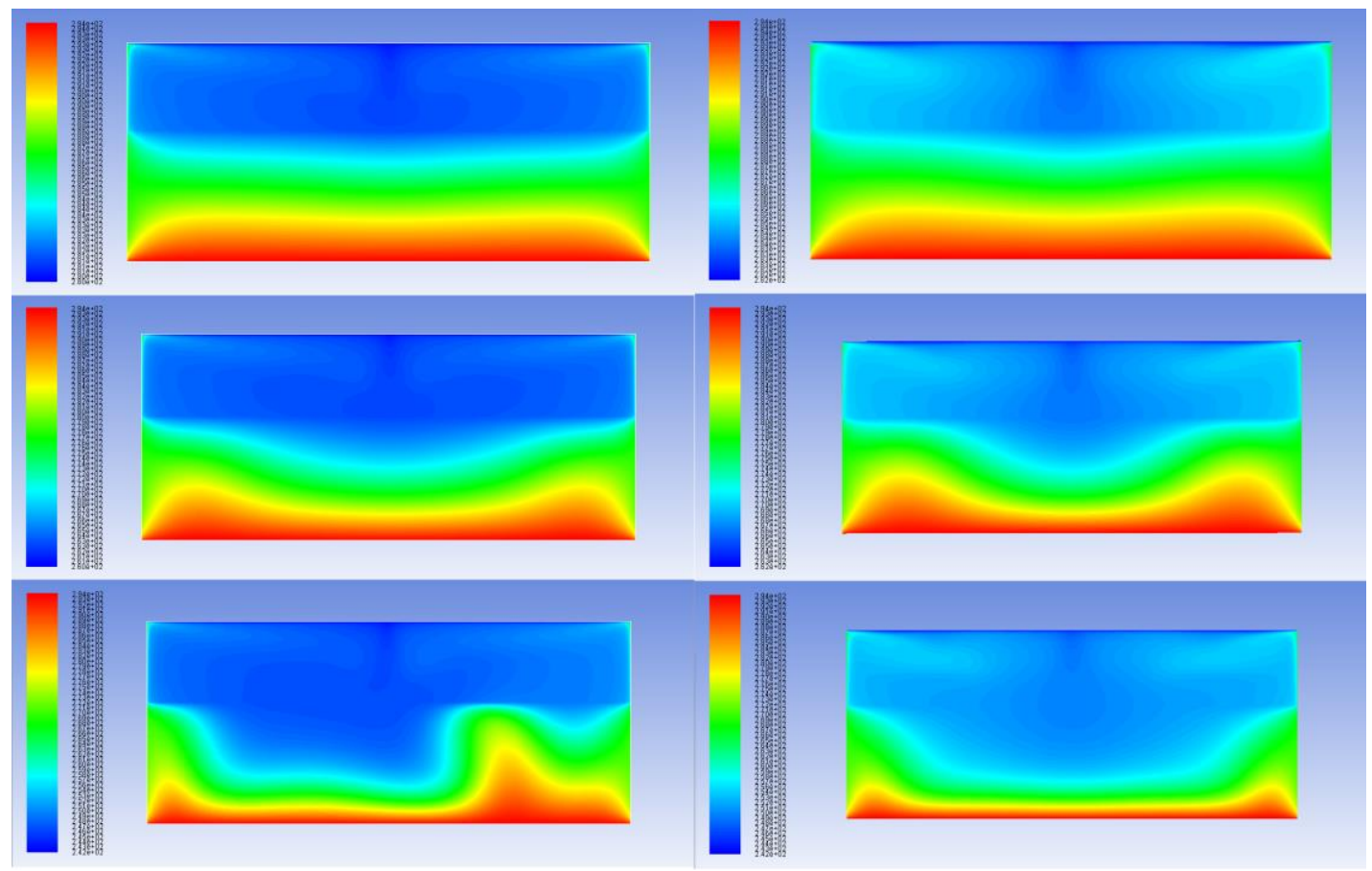

Fig. 3: Temperature contours for the two and three dimensional cases. The column to the left is for the two-dimensional simulations and the column to the right for the three dimensional simulations. The temperature contours for the three-dimensional case were taken in a vertical plane in the middle of the box parallel to its longest side. The plots are for, in order from above, 10, 30 and $50 \mathrm{~K}$ temperature difference.

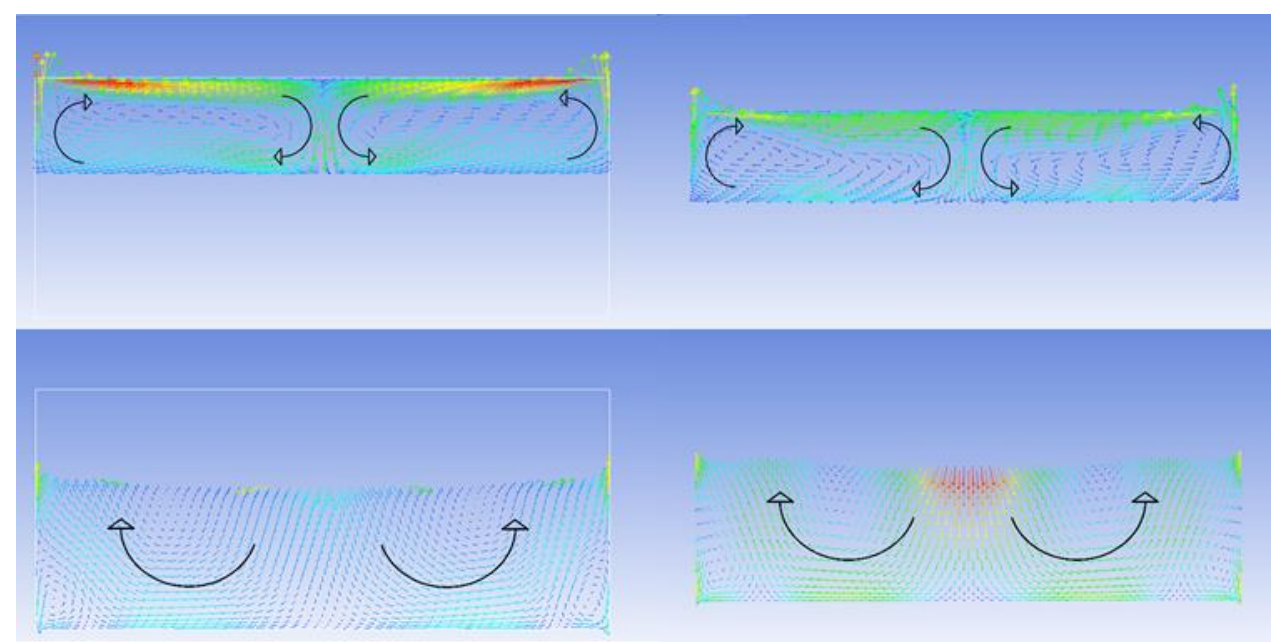

Fig. 4: Velocity vectors for the two and three dimensional simulations for the temperature difference of $30 \mathrm{~K}$. The column to the left is for the two-dimensional simulations and the column to the right for the three dimensional simulations. The first picture from above is flow in the air cavity and the second one is flow in the insulation. The arrows indicate the direction of the flow. 


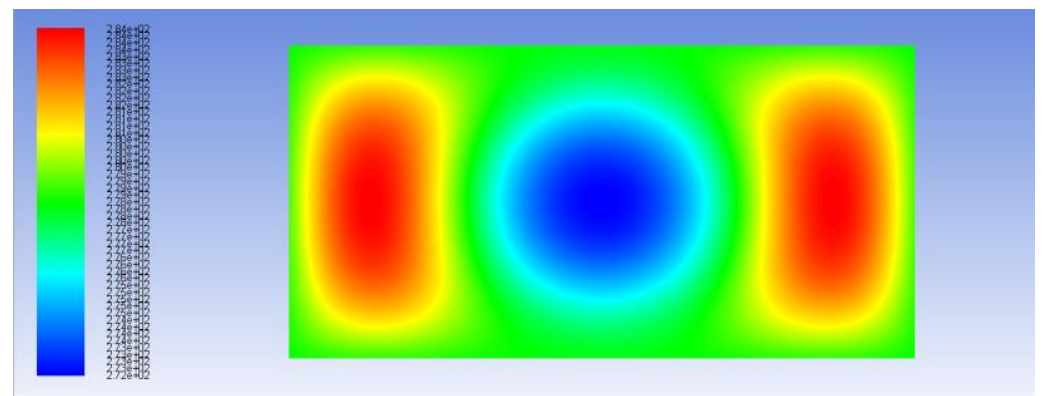

Fig. 5: Temperature contours taken in a horizontal plane in the middle of the insulation for the three-dimensional case with a temperature difference of $30 \mathrm{~K}$.

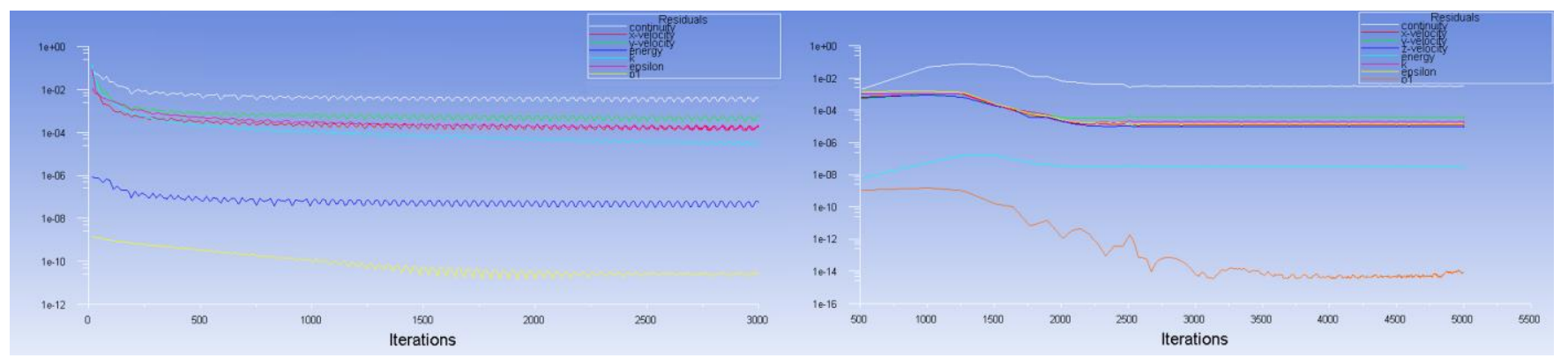

Fig. 6: Computational residuals for, in order from above, a two dimensional and a three-dimensional case at the temperature difference of $30 \mathrm{~K}$.

\section{Discussion}

As seen in Figure 2, the simulated results of the Nusselt number versus the modified Rayleigh number show a good compliance to the experimental data. The results from the two-dimensional simulations seems to be generally closer to the experimental data, while the three dimensional results gives higher Nusselt number than the experimental data and the two dimensional results for the same modified Rayleigh's number.

The velocity vector plots for $30 \mathrm{~K}$ temperature difference, see, Figure 4, shows that two distinct convection cells are formed in the air cavity in both the two and three dimensional simulations. The air motion from the convection cells in air cavity clearly penetrates into the insulation, resulting in a downwards motion in the middle of the insulation and an upwards motion at the edges of the same. The temperature contours taken in a horizontal plane in the middle of the insulation for the same temperature difference, Figure 5, indicates the same tendencies.

This air flow pattern is similar in all simulations for different temperature differences, however the magnitude of the velocities increases with the increase in temperature difference.

This phenomenon can also be used to explain the trends in the temperature contour plots, Figure 3 , where the temperature in the middle of the insulation becomes lower with increasing temperature difference between the cold and hot surface of the calculation domain. The same is due to fact that the velocities gets larger, the convection cells can more effectively transport cold air from the cold top region into the insulation.

The asymmetric disturbance for the two dimensional simulation with $50 \mathrm{~K}$ temperature difference, see, Figure 3 is probably due to the fact that the simulation is unsteady in nature, only in the case of two dimensional simulations. In general, the three dimensional simulations were found to be more stable.

Regarding the accuracy of the numerical simulations, it can be seen when looking at the picture with the residuals, Figure 6, that they are in general low and stable for both the two and three dimensional cases. The general convergence criterion in Fluent defines a converged solution when all residuals are below 10-3 except P1 and energy which have to be below 10-6 [2]. This criterion is sufficient in most situations, and is fulfilled in both the two and three dimensional simulations[3]. The highest residual is continuity in both cases but with a value between 10-2 to 10-3 even this residual is quite low. This implies that the numerical error in the simulations is low, and that the simulations should be of good quality.

As seen when comparing the simulations with and without radiation, the difference is in general quite small, but the 
addition of radiation affects the convective heat flow more in the three dimensional case than in the two dimensional one.

\section{Conclusion}

The simulations show physical results both from a visual perspective (figures for temperature and velocity distribution) and from a heat transfer perspective (Figure 2). The results obtained from computations are in agreement with the experiments. The applied method and model for porous media works well for this application. The magnitude of total rate of heat transfer is dependent on the temperature difference across the domain. The heat transfer due to radiation also plays an important role. Three dimensional computations are recommended in order to obtain an accurate picture of the flow field.

\section{Future Work}

Since the applied model for porous media works well for simulating the heat transfer in building insulation and also the buoyancy driven flow in the air cavity, more complicated parts of buildings containing insulation, for example attics or walls of buildings, can now be numerically investigated using this model.

\section{Acknowledgements}

This research work has been conducted with the help of financial and computer resources obtained from ÅForsk, ÅF Industry AB, Sweden and the Division of Building Technology, Chalmers University of Technology.

\section{References}

[1] B. Andersson, et al, Computational Fluid Dynamics for Engineers. Cambridge University Press: Cambridge, 2012.

[2] ANSYS, Inc. 2011. ANSYS FLUENT Theory Guide. ANSYS, Inc, Canonsburg, latest edition.

[3] ANSYS, Inc. 2011. ANSYS FLUENT User's Guide. ANSYS, Inc, Canonsburg, latest edition.

[4] A. Delmas, K. Wilkes, Numerical analysis of heat transfer by conduction and natural convection in loose-fill fiber glass insulation effects of convection on thermal insulation. ORNL/CON-338, 1992.

[5] A. Delmas, E. Arquis, "Early initiation of natural convection in an opened porous layer due to the presence of solid conductive inclusions," Journal of Heat and Mass Transfer, vol. 117, 1995.

[6] D. Nield, A. Bejan, Convection in Porous Media. Springer: New York, 2013.

[7] M. Serkitjis, "Natural convection heat transfer in a horizontal thermal insulation layer underlying an air layer," $\mathrm{Ph} . \mathrm{D}$. Thesis, Chalmers University of Technology, Göteborg, 1995.

[8] V. Shankar, C. E. Hagentoft, "Numerical convection in insulating porous medium," Indoor air 99, Edinburgh, 1999.

[9] V. Shankar, C. E. Hagentoft, "Influence of natural convection on the thermal properties of insulating porous medium with air cavity," Indoor air 99, Edinburgh.

[10] V. Shankar, C. E. Hagentoft, "Numerical investigation of natural convection in horizontal porous media heated from below comparisons with experiments," ASME International, Pittsburgh, 2000.

[11] V. Shankar, L. Davidson, E. Olsson, "Numerical investigation of turbulent plumes in both ambient and stratified surroundings," Journal of INDOOR AIR, Denmark, 1995.

[12] V. Shankar, L. Davidson, E. Olsson, "Ventilation by displacement: Calculation of flow in vertical plumes," ROOM VENT, Aalborg, Denmark, 1992.

[13] P. Wahlgren, "Convection in Loose-fill Attic Insulation," Ph.D. Thesis, Chalmers University of Technology, Göteborg, 2001. 